\title{
PERSPECTIVES
}

\section{Stochastic weather and climate models}

T. N. PalmeriD

Abstract | Although the partial differential equations that describe the physical climate system are deterministic, there is an important reason why the computational representations of these equations should be stochastic: such representations better respect the scaling symmetries of these underlying differential equations, as described in this Perspective. This Perspective also surveys the ways in which introducing stochasticity into the parameterized representations of subgrid processes in comprehensive weather and climate models has improved the skill of forecasts and has reduced systematic model error, notably in simulating persistent flow anomalies. The pertinence of stochasticity is also discussed in the context of the question of how many bits of useful information are contained in the numerical representations of variables, a question that is critical for the design of next-generation climate models. The accuracy of fluid simulation may be further increased if future-generation supercomputer hardware becomes partially stochastic.

Global climate models extend weather forecast models to include a more comprehensive representation of chemical and physical processes, such as those occurring in the cryosphere and biosphere. Such climate models will help society become more resilient to changes in extremes of weather and climate on longer timescales, for several reasons. Through the Intergovernmental Panel on Climate Change Working Group I reports ${ }^{1}$, such models will continue to be the primary scientific input for decisions on how fast the world economy must decarbonize to avoid the risk of increased weather and climate extremes caused by ongoing greenhouse gas emissions. In addition, because some level of anthropogenic climate change appears inevitable, climate models will play an important role at the national level in determining infrastructure investments needed to adapt societies to regional climate change as effectively as possible ${ }^{2}$. Doing this requires knowledge, as accurate as possible, on changes to the likelihood of weather and climate extremes on the regional scale. There is also a concern that 'plan B' geoengineering proposals ${ }^{3}$, such as spraying sulfuric acid droplets into the stratosphere, could detrimentally affect regional climate features such as monsoon rainfall. This can only be determined using reliable climate models. Furthermore, there is considerable interest in knowing whether specific extreme weather or climatic events can be attributed to human-induced climate change. However, such attributions depend critically on how accurately current-generation climate models simulate the circulation features associated with the types of extreme events under consideration ${ }^{4}$. In addition, irrespective of climate change, climate models play an increasingly important role in the prediction of climate variability on seasonal and decadal timescales ${ }^{5}$. The potential impact of such predictions (the predictability of which derives in large part from ocean-atmosphere coupling) is enormous. An ability to predict drought and flood reliably months or years in advance will be crucial for anticipating and mitigating crop failure ${ }^{6}$ and outbreaks of climate-related diseases, such as epidemic malaria ${ }^{7}$, or anticipating other climate-related impacts.

It is worth focusing on the notion of 'reliability'. There will always be uncertainties affecting the accuracy of any prediction that is made about the weather or climate system, no matter the timescale ${ }^{8}$. The extent to which humankind will cut its greenhouse gas emissions is uncertain, as are the computational representations of the underlying equations of climate and the observations that determine the initial conditions of a prediction. Modern-day weather forecasting uses ensemble prediction methods to estimate the impact of these uncertainties $^{8}$. In an ensemble forecast, typically 50 individual predictions are made from slightly different initial conditions. However, the spread generated in ensembles that only have initial perturbations is typically too small, particularly in the tropics, implying that the observed values fall outside the range of the ensemble too often. This implies that there is a second source of uncertainty, not represented in purely initialcondition ensembles: model uncertainty ${ }^{9}$. The representation of such model uncertainty is the topic of this Perspective.

An ensemble can be interpreted probabilistically using simple frequentism: if $40 \%$ of ensemble members predict a dry season over some region of interest, then, in the absence of model biases, the probability forecast of a dry season can be assumed to be $40 \%$. If the a priori climatological probability of a dry season is, for instance, only $10 \%$, then there may be some merit in farmers taking precautionary action by planting drought-resistant crops at the start of a growing season. However, the value to the farmer of such a decision - the seeds for which may be more expensive and produce smaller yields - depends critically on whether such probability forecasts are reliable. In this context, reliability ${ }^{10}$ requires that, over a subsample of previous ensemble forecasts in each of which the probability of a dry season is $40 \%$, then a dry season should have occurred $40 \%$ of the time. Because of model error, climate-timescale forecasts are not yet fully reliable ${ }^{11}$.

Of course, understanding how our climate system works is of great interest for its own sake, and comprehensive climate models are vital for scientific understanding. If reality can be simulated accurately with a fully comprehensive model, the essential ingredients needed to explain a particular climatic phenomenon can be discovered by removing inessential ingredients one 
by one, for example. When scientists are confident they have a good understanding of some phenomenon, it seems reasonable to suppose that communicating climate science to policy-makers and decision-makers becomes much more effective.

This Perspective discusses why, even though the underpinning equations of climate are deterministic, any computational representation of climate should be partially stochastic. The reasons for this include the nature of the symmetries of the underlying equations, the improved forecast reliability that stochastic models have over their deterministic counterparts and the implications of stochasticity for improving the algorithmic efficiency of next-generation climate models. Improving such efficiency will be vital for exploiting the benefits of exascale computing as effectively as possible.

\section{Reasons for stochastic models}

Typically, the first step in mathematically simulating a complex phenomenon is to decide how much complexity needs to be described. For a qualitative understanding of key processes at play, a low-order model with few degrees of freedom is usually sufficient. By contrast, a fully quantitative description may require a comprehensive representation of numerous processes in a computationally expensive simulator. For example, a qualitative understanding of the greenhouse effect can be obtained in a zero-dimensional energy-balance model ${ }^{12}$ in which the Earth is represented as a partial black body with specified albedo and climate variables represented by globally averaged quantities. However, a quantitative estimation of how anthropogenic greenhouse gas emissions will increase the temperature of the Earth's surface requires representation of the climate as comprehensively as possible as a timeevolving 3D system. Between these extremes exists a hierarchy of climate models.

The notion of stochasticity was first introduced ${ }^{13}$ into climate models in the most idealized end of the hierarchy. In such models, the ocean-land-cryosphereatmosphere system is treated as a zerodimensional energy-balance system in which high-frequency weather fluctuations are represented by white stochastic noise and the ocean-land-cryosphere system is simply a slowly varying component of the climate system. The coupling of the ocean-land-cryosphere system to the atmosphere reddens the stochastic weather noise, producing stochastic low-frequency climate fluctuations. There is still debate in the ocean-modelling community about the extent to which this is the dominant role of the ocean in the climate system ${ }^{14,15}$.

The value of stochasticity in such idealized low-order stochastic models is well established. However, there is debate as to the extent to which stochasticity should play a role at the opposite end of the modelling hierarchy, in ab initio climate models that attempt to solve the underpinning laws of physics for the climate system as comprehensively as possible. I maintain ${ }^{16}$ that stochasticity should play a vital role in all models, no matter how comprehensive they aspire to be.

The primitive laws of climate physics describe the evolution of conserved quantities such as energy, momentum and mass. For example, conservation of momentum in a continuum fluid such as the atmosphere or oceans is described by the well-known Navier-Stokes partial differential equations. Not only has it not, in general, been possible to solve these equations exactly but also even basic mathematical properties of these equations, such as uniqueness of solutions, are unknown ${ }^{17}$. Hence, finite numerical methods must be used to solve such equations approximately. However, even with the biggest supercomputers to date, the equations are projected on numerical grids that are typically $100 \mathrm{~km}$ in the horizontal for global climate models and $25 \mathrm{~km}$ for global weather forecast models. These grids are many orders of magnitude larger than the smallest viscous scales represented in the Navier-Stokes equations.

There are many important processes in the global climate system that cannot currently be represented by numerical solutions of the Navier-Stokes equations truncated on a grid of $25 \mathrm{~km}$ or larger. Examples include all cloud systems - including deep convective
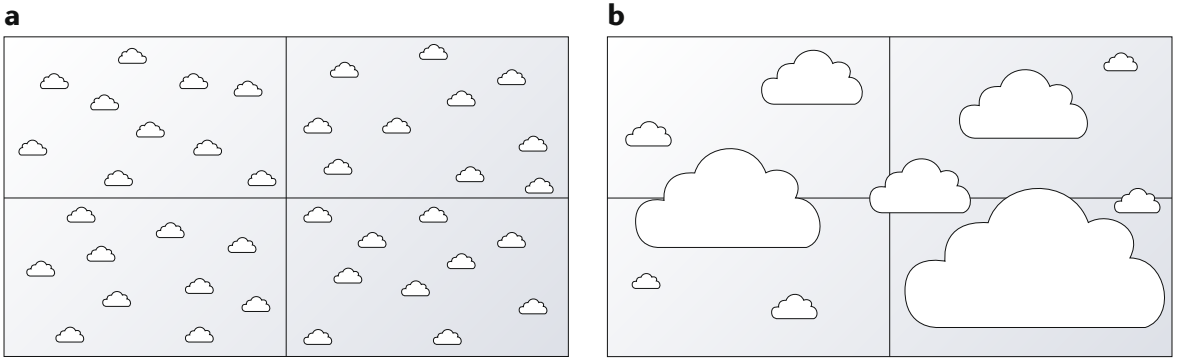

Fig. 1 | Deterministic parameterization is justified only when there is scale separation between resolved and unresolved flow. a | Schematic of a hypothetical situation in which there is some scale separation between resolved flow (at the scale of the grid) and unresolved flow (clouds that are substantially smaller than the grid), justifying the notion of deterministic parametrization. $\mathbf{b}$ | Schematic of the more realistic multiscale situation (consistent with the scaling symmetries of the underlying equations) in which there is no clear scale separation between resolved and unresolved flow, justifying the notion of stochastic parametrization.

cumulonimbus-type clouds, which play a crucial role in transporting heat, moisture and momentum vertically in tropical latitudes - orographic gravity waves and ocean mesoscale eddies. Historically, these unresolved processes are represented by semi-empirical formulae, known as parameterizations ${ }^{18-20}$. Such parameterizations have conventionally provided a deterministic estimate of the bulk effect of subgrid-scale processes on the grid-scale flow. By construction, such estimates are deterministic functions of the larger grid-scale flow, motivated by ideas in statistical mechanics. As such, the validity of deterministic parameterization depends on an assumption that the relevant subgrid processes are in some local quasiequilibrium with the resolved-scale flow. However, such an assumption rarely holds in practice ${ }^{21}$. For example, in convectively active regions, instead of finding a large number of quasi-independent small-scale cloud systems (FIG. 1a), it is frequently found that convective clouds aggregate and in so doing generate a spectrum of scales (FIG. 1 b).

Although the Navier-Stokes equations cannot be solved directly, they have certain symmetry properties that should be respected in numerical solutions of the equations, as far as possible. One of these is a scaling symmetry ${ }^{22}$ if $u(x, t)$ is the velocity field and $p(x, t)$ is the pressure field associated with a solution to the Navier-Stokes equations, then so are

$$
u_{\tau}(x, t)=\tau^{-\frac{1}{2} u}\left(\frac{x}{\tau^{1 / 2}}, \frac{t}{\tau}\right)
$$

and

$$
p_{\tau}(x, t)=\tau^{-1} p\left(\frac{x}{\tau^{1 / 2}}, \frac{t}{\tau}\right)
$$


where $\tau>0$ is a dimensionless scaling parameter. Such scaling symmetry provides the basis for the self-similar nature of fluid turbulence and is consistent with the ubiquitous existence of power-law behaviour ${ }^{23,24}$ in multiscale observations of the atmosphere and oceans (FIG. 1 b).

Imposing a numerical scheme with a hard truncation limit (with subgrid processes as deterministic functions of the large-scale flow) violates the scaling symmetry of the Navier-Stokes equations. It might be imagined that the impact of such violation is unimportant on scales that are large compared with the truncation scale. However, this need not be so. Let $E(k)$ denote the kinetic energy in the atmosphere per unit wavenumber at horizontal wavenumber $k$. At large scales at which the Earth's rotation plays a dominant role, $E(k)$ satisfies the power $\operatorname{law}^{24} E(k) \sim k^{-3}$. However, on scales smaller than about $100 \mathrm{~km}$, the energy power law shallows to $E(k) \sim k^{-5 / 3}$. Let $\tau\left(k_{\mathrm{L}}\right)$ denote the timescale associated with variability in the large-scale flow at wavenumber $k_{\mathrm{L}}$. Then, according to simple scaling arguments ${ }^{17}$, on timescales greater than $\tau\left(k_{\mathrm{L}}\right)$ errors in large-scale variability can be sensitive to errors in small-scale components of the flow. This argument indicates that minimizing systematic errors in the representation of large-scale processes requires minimization of systematic errors in the representation of small-scale processes. One systematic error in the representation of the small scales is to treat them as 'slave' to the large scale, as is done in conventional deterministic parameterization. A way to alleviate such a systematic error is to treat the unresolved scales as partially stochastic.

Consistent with this failure to respect the scaling symmetries of the Navier-Stokes equations, conventional deterministic climate models exhibit substantial systematic biases when compared with observations ${ }^{25,26}$. Despite many decades of work by scientists in weather and climate institutes around the world, the biases are still substantial in magnitude, and often comparable to the signals that such models attempt to predict or simulate. There have been numerous attempts over the years to improve deterministic parameterizations to try to eliminate such biases. Progress has been slow ${ }^{27}$.

\section{Stochasticity in practice}

Deep convective clouds are a consequence of thermally induced instabilities. However, their precise structures arise from a complex melange of processes, which affects all variables in the whole atmospheric column from the Earth's surface to the top of the troposphere and, indirectly, to higher levels still. In credible parameterized representations of convective clouds in a climate model, the model parameterizations for deep convection, cloud cover, longwave and shortwave radiation and boundary-layer turbulence act together in a balanced holistic way. Therefore, stochastic generalizations of such parameterizations must avoid breaking this holistic balance too much, such as would happen if one parameterization were perturbed completely independently of another. This notion strongly motivated the development of the first stochastic parameterization to be implemented into a comprehensive operational weather and climate model: the stochastically perturbed parameterized tendency (SPPT) scheme ${ }^{28,29}$. The SPPT scheme perturbs the sum of the parameterized tendencies:

$$
\frac{\partial X}{\partial t}=D+(1+e) \sum_{i} P_{i}=D+(1+e) P
$$

where $X$ stands for a prognostic variable (such as temperature, fluid velocity or moisture), $D$ denotes the advective tendency (that is, time rate of change) from the explicitly resolved model dynamics and $P_{i}$ denotes the tendency from the $i$ th (deterministic) parameterization scheme. In the current form of the SPPT scheme ${ }^{29}$, the stochastic perturbations $e$ are produced from a spectral pattern generator, based on the sum of three independent horizontal fields with correlation scales of $500 \mathrm{~km}, 1,000 \mathrm{~km}$ and $2,000 \mathrm{~km}$. The fields are evolved with three (first-order) Markov processes with temporal autocorrelation times of 6 hours, 3 days and 30 days, and standard deviations of $0.52,0.18$ and 0.06 , respectively. The smallest-scale perturbations are the most important for medium-range forecasts, whereas the longer-scale perturbations are more important on monthly and longer timescales. In addition to the holistic assumption above, the SPPT scheme is underpinned by the notion that the larger the parameterized tendency $P$, the larger the stochastic forcing term. That is, it is assumed that the applied noise is multiplicative.

The SPPT scheme adds inherent variability to the subgrid parameterizations so that they are no longer slave to the resolved scales. In addition, spatiotemporal autocorrelations of the noise introduce stochasticity directly onto scales larger than the truncation scale. In this way, the SPPT scheme 'blurs' out the notion of a hard truncation scale and makes the resulting numerical representations more consistent with the scaling symmetries above.
One objection to the SPPT scheme is that it appears to be a 'bolt-on' extra to a set of previously developed deterministic parameterizations, whereas parameterizations should be developed stochastically ab initio ${ }^{30-32}$. In fact a number of approaches that are stochastic a priori have been developed for individual parameterizations ${ }^{33-37}$ and reviewed elsewhere $^{38}$. Another objection to the SPPT scheme is that it does not obviously respect conservation properties. Work is currently ongoing to ensure that it does respect global conservation of moisture in particular. Also, the SPPT scheme is not a complete representation of stochastic subgrid processes. One missing process is that of stochastic 'backscatter ${ }^{39-41}$. For example, in convective backscatter schemes, it is assumed that the kinetic energy associated with sufficiently well-organized convective cloud systems projects stochastically onto resolved-scale balanced circulations. A number of operational weather prediction models include both an SPPT scheme and backscatter representations.

To date, the development of all parameterizations has been based around a combination of data, theory and human creativity. This has made the development of parameterizations subjective. Questions that arise include how to test the assumptions in the SPPT scheme and justify the chosen values for the stochastic parameters; how to develop stochastic parameterizations ab initio; and how to make the development of stochastic parameterization more objective.

Stochastic parameterization is now a productive meeting ground for theoreticians and operational weather and climate modellers ${ }^{42}$. An example of a more theoretical approach to stochastic parameterization is that based on MoriZwanzig projector operator theory. This theory is well established in statistical physics, and has emerged in recent years in climate modelling and is now bearing practical fruit. The Mori-Zwanzig projection operators act in phase space and project onto a subspace of 'slow' phase-space functions. In the present context, slow refers to quantities resolved by the model. This approach allows the Liouville equation to be projected onto slow and fast subspaces, enabling a reduced model to be formulated in the form of a generalized Langevin equation. The theory is implicit, that is, it does not provide the means to find these slow variables from a given Hamiltonian. Despite this, Mori-Zwanzig theory has been exploited for proposing explicit formulas for parameterizations in climate models $s^{43-45}$. 
Stochastic parameterizations constructed using the Mori-Zwanzig theory have the potential to be well suited to a formulation in which the parameterization is aware of the physical scale of the grid box it is operating inside ${ }^{46}$, possibly useful in the so-called grey zone in which processes are neither fully resolved nor fully parameterized and in which memory effects could be relevant. Indeed, Mori-Zwanzig theory has motivated a recent stochastic parameterization scheme using cellular automata to represent unresolved convective cloud systems ${ }^{47}$. The scheme is designed for the US National Oceanographic and Atmospheric Administration's next-generation global prediction system. In the proposed scheme, the need for a dynamical memory term, a key feature of the Mori-Zwanzig formalism, is emphasized.

One method for testing stochastic parameterizations is through a technique known as coarse graining ${ }^{48-50}$. In this technique, a high-resolution model, such as a limited-area cloud resolving model, is treated as 'truth'. The high-resolution fields and tendencies are then averaged to the resolution of the target weather and climate model and fed into the model's parameterized tendencies. In this way, it is possible to compare the true tendencies and the parameterized subgrid tendencies of the coarse-grained grid. In these studies (notably REF. ${ }^{50}$ ), the multiplicative form of the noise term in the SPPT scheme was supported by coarse-graining analysis, but the linear dependency on $P$ was found to be an oversimplification. Coarse graining has also provided justification for the spatiotemporal characteristics of the applied noise. However, coarse graining suggest ${ }^{50}$ that the notion that the applied stochasticity should be completely coherent across all processes and variables may also be an oversimplification. For example, little coherence is expected between convective and orographic parameterizations. A method for perturbing away from the fully holistic structure of the SPPT scheme has been developed ${ }^{51}$ with promising results. In addition to this, a rather general theoretical basis for stochastic parameterization has been developed based explicitly on the notion of coarse graining, using techniques in stochastic geometric dynamics ${ }^{32}$.

Output datasets from high-resolution models are large and analysing information from them for developing parameterizations is challenging. Recent developments in the field of artificial intelligence provide a way forward. Representing parameterizations by probabilistic neural nets not only provides a way to develop stochastic parameterizations objectively but also speeds up computation time considerably. This is a key problem for the quest to develop in the first half of the next decade a global climate model that can resolve deep convective cloud systems (discussed below). At present, parameterizations can take $50 \%$ of the computational time. Considering how uncertain these parameterizations are, this expense can hardly be justified. The possibility of representing outgoing longwave radiation with a neural net, trained on output from an operational parameterization, has already been demonstrated ${ }^{52,53}$. Generalizing this approach to describe all the parameterizations (and elements of Earth-system complexity) is likely to be a real growth area for weather and climate modelling in the coming years. However, based on the discussion above, it is vital for such artificial intelligence-based schemes to be inherently stochastic in nature.

Although the discussion above has focused on stochastic parameterization in the atmosphere, the concept is also relevant in other parts of the Earth system, such as the oceans ${ }^{54,55}$, land surface ${ }^{56}$, sea ice ${ }^{57}$ and air-sea fluxes ${ }^{58}$.

\section{Impact of stochasticity}

The most direct and immediate impact of stochastic parameterization is on the reliability and the skill of probabilistic weather forecasts, which can be measured using the continuous rank probability skill score (CRPSS) (FIG. 2). CRPSS is closely linked to the notion of potential economic value ${ }^{59}$. This link is based on a simple decision-theoretic model in which a user must decide when to take precautionary action, at $\operatorname{cost} C$, to eliminate a preventable loss $L$ that would occur if some weather event $E$ were to occur without precautionary action being taken. In this idealized framework, the user should take precautionary action when $C<p L$, where $p$ is the forecast probability of $E$. The CRPSS is an integrated measure of the value of such decisions, for all possible users and all possible events ${ }^{60}$. The impact of stochastic parameterization on CRPSS - and hence potential economic value - is dramatic in the tropics (FIG. 2), where parameterized processes have a large impact on the largescale flow. Stochastic parameterization enables a forecast at 5 days to have the skill of a forecast at 2 days without stochastic parameterization. Given that it typically takes 10 years of pooled effort of an institute

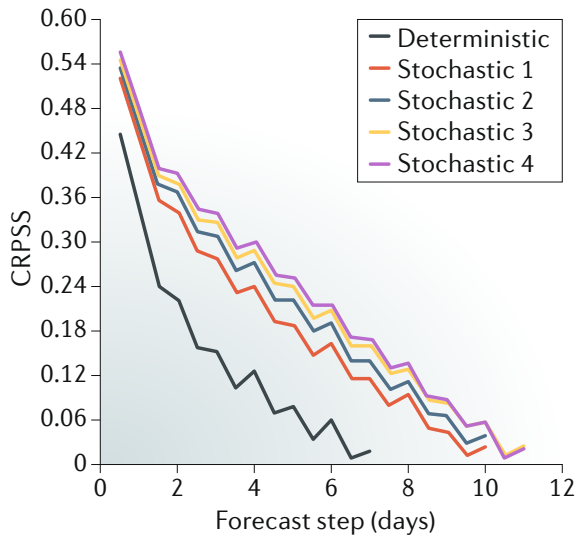

Fig. 2 Impact of stochastic parameterization on continuous rank probability skill scores for temperature on the $850 \mathrm{hPa}$ pressure surface in the tropics. The grey line is for an ensemble forecast made with a conventional deterministic model. The coloured lines are for ensemble forecasts with additional stochastic parameterization, with different flavours of stochasticity. CRPSS, continuous rank probability skill score. Reproduced with permission from REF. ${ }^{29}$, copyright ECMWF.

to improve deterministic forecast skill by just 1 day, this is certainly a substantial impact.

In this example, the positive impact of stochastic parameterization is straightforwardly attributed to the fact that stochastic parameterization represents model uncertainty explicitly. Doing so increases the spread of the ensemble. Hence, the number of occasions in which reality lies outside the range of the ensemble is decreased. However, it has been argued above that stochastic parameterization is more than a way to represent model uncertainty - it is a way for the numerical representations of the underlying equations of motion to avoid violating one of the underpinning symmetries of these equations of motion. This means that stochastic parameterization can be expected to have a positive impact on model systematic biases when compared with observations.

Indeed, the SPPT scheme has a notable impact in reducing systematic errors in the strength of the simulated seasonal-mean trade winds and tropical precipitation fields and in the strength of the simulated intraseasonal Madden-Julian oscillation ${ }^{61,62}$. On multi-year timescales, stochastic parameterization can have a dramatic impact on simulations of the coupled ocean-atmosphere El Niño phenomenon (FIG. 3). In a deterministic climate model in which the El Niño had too strong a spectral peak at around 2-4 years, introducing stochastic parameterization produces a far flatter spectral response on the interannual 

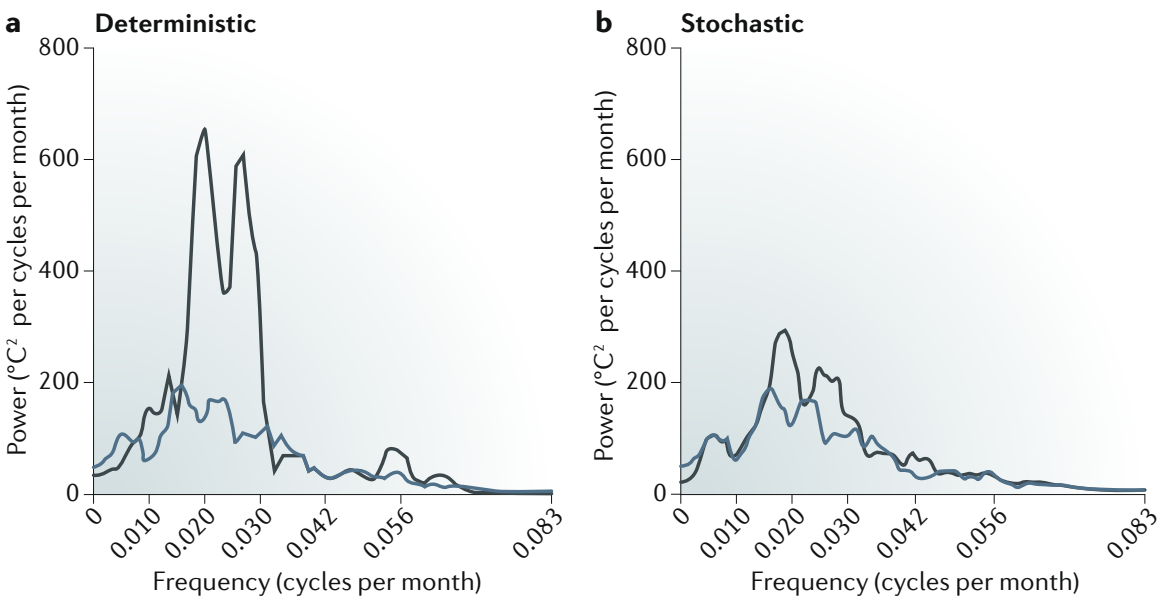

Fig. 3 | Power spectra of average sea surface temperature in the Niño-3.4 region in 135-year-long simulations using the National Center for Atmospheric Research Community Atmosphere Model coupled to an ocean model. a | Compared with observations from the Hadley Centre Sea Ice and Sea Surface Temperature dataset (light blue), the simulation (dark blue) has three times more power for oscillations with periods between 2 and 4 years. $\mathbf{b} \mid$ When the simulation is repeated with the stochastically perturbed parameterized tendency scheme, the temperature variability in this range is reduced, leading to a better agreement between the simulated and observed spectra. Reproduced with permission from REF. ${ }^{63}$, AMS.

timescale ${ }^{63}$. In turn, this flattening has a profound impact on the modelled monsoon variability ${ }^{64}$. In some ways, this is a remarkable result because the intrinsic timescale of the imposed stochasticity is much faster than the characteristic timescale for El Niño. This indicates that there are some mechanisms at play that allow such stochasticity to cascade to longer timescales. It is likely that this cascade involves the impact of convection on the Madden-Julian oscillation on intermediate subseasonal timescales ${ }^{63}$.

The SPPT scheme also has a profound impact on the ability of a model to simulate quasi-stationary weather regimes ${ }^{65}$ in the Northern Hemisphere extratropics. To illustrate the relevance of this impact, consider one of the most striking large-scale climate anomalies of 2018. In September 2018 , the Met Office announced ${ }^{66}$ that the summer of 2018 was the warmest on record in England. Indeed, the summer period saw heatwaves in a number of regions around the Northern Hemisphere ${ }^{67}$. Such warmth is consistent with the long-term increase in global mean temperature due to anthropogenic greenhouse gas emissions and one real-time attribution study ${ }^{68}$ concluded that these heatwaves were made twice as likely by anthropogenic emissions.

How certain are such attribution statements? The proximate cause of such heatwaves was a relatively weak and oscillatory jet stream, where the wave phase was geographically fixed over much of the summer season. Consistent with this, crop yields reduced dramatically ${ }^{69}$ in regions affected by heatwaves, indicating a concomitant decrease in rainfall, and the energy produced by wind farms also decreased ${ }^{70}$, indicating a concomitant decrease in wind speed. Therefore, in attempting to attribute such heatwaves to climate change, a crucial question is whether greenhouse gas emissions are making these persistent wavy-jet regimes more likely. A potentially straightforward way to answer such a question would be to study in the model integrations the change in the frequency of occurrence of persistent wavy jets under increased levels of $\mathrm{CO}_{2}$. There is no obvious signal in the models. However, it is difficult to draw a robust conclusion from this result because such long-lived quasi-stationary anomalies are severely under-represented in climate models ${ }^{71}$. This problem is most clearly seen - and most widely studied - in the context of long-lived blocking anticyclones in winter, but is also apparent in summer. Because of these model deficiencies and the fact that climate is a highly nonlinear system so that biases cannot be removed by simply subtracting systematic errors from output fields, it is not currently possible to rigorously attribute such heatwaves to climate change. Therefore, any quantitative estimates of the impact of anthropogenic climate change should be treated with caution.

One reason for the under-representation of quasi-stationary anomalies is that the resolution of climate models is inadequate, even though resolution increases over the past few years have improved matters ${ }^{72}$. There are two reasons why resolution is important. The first is that poor resolution means that the topographic forcing of quasi-stationary Rossby waves is too weak. A second reason is linked to transient eddy activity. It has been known since studies of the persistence of the 1976 heatwave over the $\mathrm{UK}^{73}$ that long-lived anticyclonic anomalies are maintained by transient eddies, in the form of mobile weather systems originating over the Atlantic and steered north and south of the anticyclone by the associated wavy jet in the upper troposphere.

If transience is important, the effect may help with stochasticity. The geopotential height anomalies associated with four dominant circulation regimes in winter over the Euro-Atlantic sector are shown in FIG. 4a-d. When the operational model from the European Centre for Medium-Range Weather Forecasts is run at typical climate resolution, it poorly represents the structure of such regimes and underestimates their persistence ${ }^{74}$. Increased resolution improves the simulations considerably. However, much of the impact of increased resolution could be simulated using stochastic parameterization instead. Both the highestresolution deterministic model and the medium-resolution model with stochastic parameterization have statistical significance similar to the observations (FIC. 4e).

The fact that noise can improve the ability of models to simulate regime behaviour can be demonstrated in much simpler models. Consider, for example, the iconic Lorenz 63 model ${ }^{75}$ in which independent stochastic noise terms $\eta_{i}$ have been added to each of the original (deterministic) equations of motion:

$$
\begin{aligned}
& \dot{X}=-s X+s Y+\sigma \eta_{1} \\
& \dot{Y}=-X Z+r X-Y+\sigma \eta_{2} \\
& \dot{Z}=X Y-b Z+\sigma \eta_{3}
\end{aligned}
$$

where $s$ is the Prandtl number, $r$ is the rescaled Rayleigh number and $b$ is a parameter depending on the system size and periodicity. For a range of noise amplitudes $\sigma$, stochasticity stabilizes the regimes ${ }^{76}$ (FIG. 5). Similar behaviour can be found in idealized nonlinear models that are closer to the atmospheric equations of motion, such as the Charney-Devore model ${ }^{77}$. From a dynamical systems perspective, this surprising impact can be understood by considering the outset of an unstable fixed point corresponding to the centroid of the regime $^{78}$. This outset typically is confined to a small region of state space and motion along the outset is disrupted by the presence 


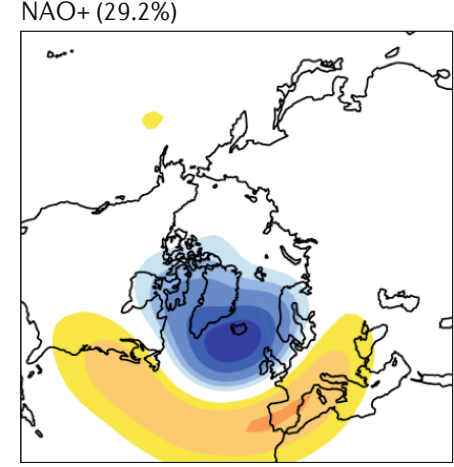

c $\operatorname{AR}(22.8 \%)$

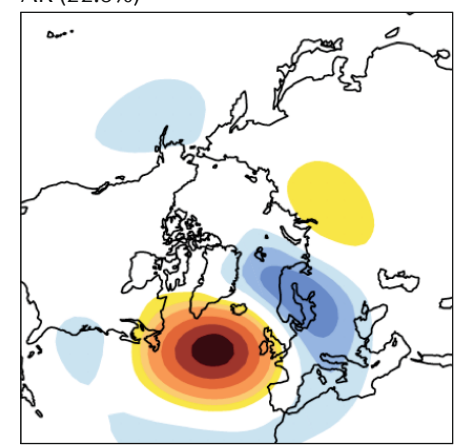

b

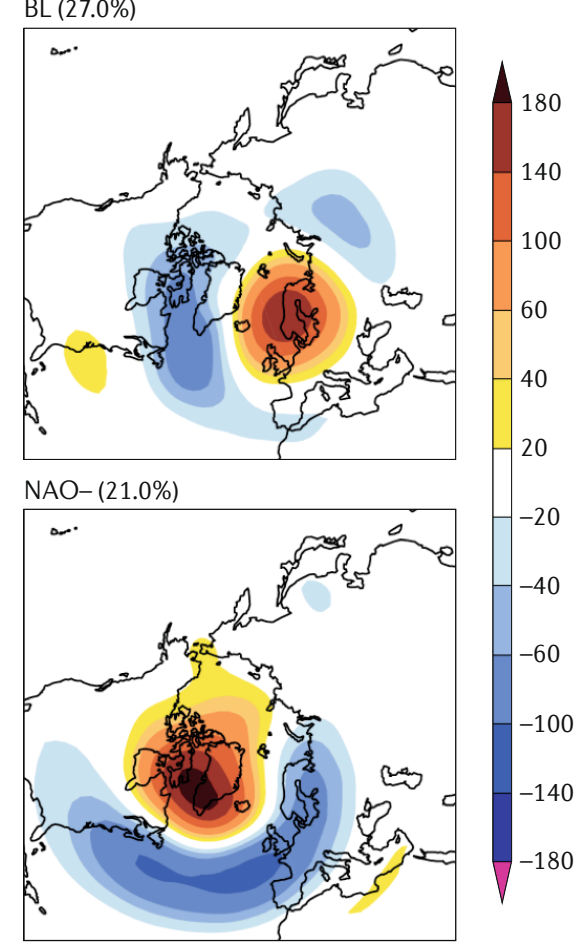

e

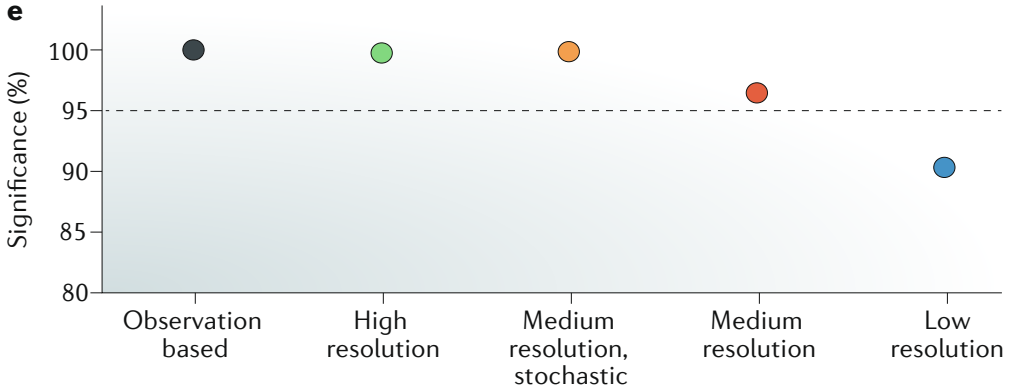

Fig. 4 Quasi-stationary circulation regimes over the European-North Atlantic domain and their statistical significance. a | Positive North Atlantic Oscillation (NAO+) cluster. b | European blocking (BL) cluster. c | Atlantic ridge (AR) cluster. d | Negative North Atlantic Oscillation (NAO-) cluster. The climatological frequency of occurrence for each cluster in panels $\mathbf{a}-\mathbf{d}$ is given next to the name. e | Statistical significance of the regimes shown in panels a-d, determined by comparing inter-regime distance with intra-regime standard deviation, averaged over all four clusters. The observation-based estimate of significance is the standard to which the other points are compared. The other points refer to output from a version of the European Centre for Medium-Range Weather Forecasts model when the model is run at different spectral resolutions - medium-resolution simulations with stochastic parameterization are comparable to high-resolution deterministic simulations. Panels a-d are reproduced from REF. ${ }^{74}$, Springer Nature Limited. Panel e data from REF. ${ }^{74}$.

of stochastic noise, keeping the state in the regime longer than it would otherwise stay. It remains to be seen to what extent this dynamical systems analysis is consistent with more meteorological analyses based on the interaction between transient eddies and the time-averaged mean flow.

In this example, the regimes already exist in the deterministic Lorenz model and the noise merely strengthens them. However, in the more complex Lorenz 96 model $^{79}$, a deterministically parameterized truncation of the small-scale variables in this model completely fails to simulate one of the regimes of the full model, whereas the stochastically parameterized version of the model captures this regime well ${ }^{80}$. This result accords with the results discussed above.

In models with insufficiently strong regime structure (a situation comparable to overly shallow potential wells in state space), there is the potential for ensemble members to become too spread out - the opposite of the more generic situation of underdispersive ensembles in the tropics. Indeed, there is evidence that seasonal forecasts of the North Atlantic Oscillation a large-scale pressure pattern over the North
Atlantic region - are overspread in this sense ${ }^{81,82}$. As discussed above, a cure for this problem should be found in increased model resolution and stochastic parameterization.

\section{More accuracy with less precision}

The results described above suggest that a climate model with resolution of $25 \mathrm{~km}$ and with stochastic parameterization may be a reasonable compromise for some climate studies in the coming years. With a grid too much coarser, the topography is not well resolved; with a grid too much finer, the model resolution enters the 'grey zone' in which key processes are neither well resolved nor well parameterized. Consistent with results discussed above, it has been found that $25 \mathrm{~km}$ models with stochastic parametrization produce more realistic numbers of tropical cyclones compared with corresponding deterministic models. (P.-L. Vidale and A. Weisheimer, personal communication). More realistic simulations of tropical cyclone numbers are possible by further increasing model resolution. However, including stochastic parameterization is certainly the cheaper of the two options.

However, although a stochastic $25 \mathrm{~km}$ model has reduced biases compared with a deterministic model, stochasticity certainly does not eliminate such biases completely. For the reasons discussed in the introduction, it is vital to develop a model in which these biases are small compared with the signals the model is intended to predict. This goal can be framed more qualitatively in terms of a 'climate Turing test' ${ }^{27}$ : for scales well resolved by a climate model, it should be difficult to tell whether one is looking at fields from the model or from the real world. Because of systematic errors, this test has not yet been passed.

For this reason, there is a need to develop models with much finer grids than those used at present ${ }^{83}$. A $1 \mathrm{~km}$ grid (with commensurate vertical resolution) represents an important goal because such a grid allows three key parameterizations - deep convective clouds, orographic gravity waves and ocean mixing by mesoscale eddies - to be eliminated and these processes instead be represented by the laws of physics. Of course, such a procedure will not eliminate the need for stochastic parameterizations. Even with a $1 \mathrm{~km}$ grid, key processes such as subgrid turbulence, the effects of aerosols and cloud microphysics need to be parameterized and the general arguments above still apply. However, it can be expected that many of the endemic large-scale model systematic errors that have plagued climate models will finally be substantially reduced once these 
key parameterizations have been eliminated, allowing the climate Turing test to finally be passed.

However, running a global model over climate timescales with a $1 \mathrm{~km}$ grid is prohibitively expensive with current petascale computing technology. Even with dedicated next-generation exascale supercomputers, such a model is only barely feasible, and substantial improvements in computational efficiency will also be needed if the 1-km-grid model is to be a realistic goal with the advent of exascale technology in the next decade.

In this regard, stochasticity brings an additional benefit: it allows more computationally efficient code to be developed. For example, subgrid parameterizations require stochasticity, and the impact of parameterizations percolates upscale in the dynamical core during integrations. Therefore, a valid question is whether the default 64-bit double-precision floating-point representations of variables are needed in a weather or climate model ${ }^{84}$. Why not 32-bit or even 16-bit precision? Ensemble weather forecasts with almost all variables represented with 32-bit floating-point reals shows no degradation in skill and a $40 \%$ reduction in computational time to solution ${ }^{85}$. There is substantial evidence that in fact many of the variables could be represented with 16-bit floating-point reals ${ }^{86-88}$.

Indeed, the market for artificial intelligence has driven graphics processing unit (GPU) manufacturers to produce GPUs that are capable of efficiently executing arithmetic operations using 64-bit, 32-bit or 16-bit floating-point numbers. Representing variables, when possible, with 32 bits or 16 bits rather than 64 bits can make a big difference, not only in the sheer number-crunching load, but also in the amount of data that are transported inside a supercomputer from processor to processor and memory to processor. As most of the energy needed to power a present-day supercomputer is used to transport data, restricting the data flow to that which contains useful information ${ }^{89}$ is critically important. One of the benefits of a spectral climate model (in which the equations of motion are projected onto finite-dimensional spherical harmonics) is that its basis functions are scale dependent. Therefore, in such a framework, it is straightforward to design a numerical scheme with scale-aware numerical precision: the higher the total wavenumber associated with a particular spherical harmonic, the lower the numerical precision needed in the corresponding evolution equation for that scale. Ideally,
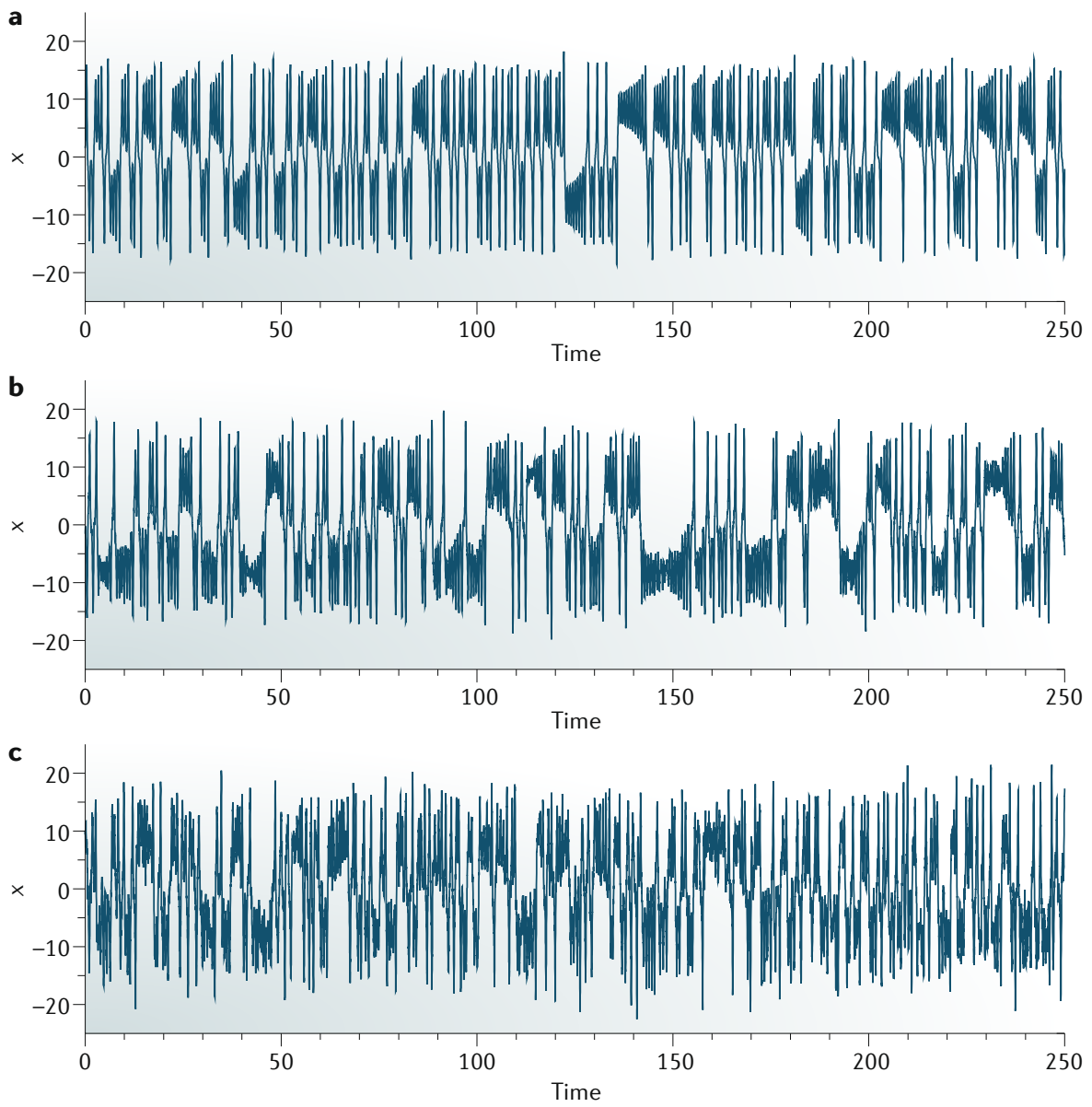

Fig. 5 | At intermediate noise levels, the typical regime residence times for the time series for the x component of the Lorenz 63 system shift to larger values. The noise levels $\sigma$ are $\sigma=0$ (panel a), $\sigma=3.2$ (panel $\mathbf{b}$ ) and $\sigma=8$ (panel $\mathbf{c}$ ). The Lorenz 63 system is given in equation 4. Reproduced with permission of The Royal Society, from Enhanced regime predictability in atmospheric low-order models due to stochastic forcing, Kwasniok, F., A372, 2014 (REF. ${ }^{76}$ ); permission conveyed through Copyright Clearance Center, Inc. RSC.

precision would be a parameter that can be varied more continuously with scale than the powers of two discussed here allow ${ }^{90,91}$. Field-programmable gate array computers allow such variable precision ${ }^{92}$.

This discussion of computational efficiency raises an important issue. Typically, the dynamical core and sets of parameterizations are developed by different groups of scientists and software engineers. The development of the dynamical core is typically the domain of specialists in numerical analysis. For example, such analysts may explore new types of numerical schemes or investigate the impact of increasing the level of numerical accuracy of the spatial or temporal discretization schemes. Such new types of numerical scheme can have advantages in terms of formal accuracy, albeit typically at some computational cost. However, a key question is whether these advantages remain after the uncertain parameterization schemes have been included. The problem is that because such parameterization schemes are themselves computationally expensive, it is inconvenient and inefficient to have to couple the parameterizations into the dynamical core in the initial development phase of a new numerical scheme. For this reason, a simple additive stochastic dataset has been proposed ${ }^{93}$, based on the mean characteristics of the SPPT scheme above, for use when testing dynamical cores.

In contemporary or next-generation supercomputers, the value of low-precision modelling is typically considered to be less about increasing the number of floatingpoint operations per second (FLOPS), and more about reducing the number of bits that are transported. However, restricting data transport to those bits that have useful information is not the only benefit that can come from low-precision computing. The well-publicized end of Dennard scaling 


Glossary
Albedo
A non-dimensional measure of the diffuse reflection
of sunlight from a surface. More specifically, the ratio of
radiosity to irradiance.
Biosphere
The regions of the Earth system occupied by living
organisms.
Cryosphere
Those portions of Earth's surface where water is in solid
form.
Madden-Julian oscillation
A planetary-scale eastward-propagating oscillation in
surface pressure (and related variables), primarily
located in the tropics, with a periodicity of about
30-60 days.
Orographic
Related to the topography of mountains.
Outset
In dynamical systems theory, a saddle-point instability
has both a stable and unstable manifold in state space,
unstable manifold.

Rossby waves

Planetary-scale wavelike disturbances in surface pressure (and related variables) whose existence and properties are dependent on the rotation of the Earth.

\section{Skill}

A measure of the practical value of weather forecasts. For probabilistic forecasts, the skill combines the reliability of the forecast probabilities and the sharpness of the forecast probability distributions.

reflects the fact that no matter how small a transistor shrinks, there is a power baseline needed for it to operate deterministically. Because of this, transistor density cannot be increased indefinitely because eventually the heat generated would melt the components. However, as discussed above, not all transistors need to operate deterministically to perform stochastic computations. Hence, transistor density can perhaps be increased if the requirement is only for probabilistic rather than strict deterministic output. If processor $P_{1}$ performs $F_{1}$ exact FLOPS and processor $P_{2}$ performs $F_{2}>F_{1}$ inexact FLOPS (particularly in relation to the higher-order mantissa bits of floating-point variables), it could be that the inexact processor enables more accurate predictions ${ }^{94,95}$, because the higher FLOP rate could allow for an increase in model resolution or ensemble size, and the additional inexactness would be within the bound of uncertainty associated with the inherent stochasticity of the subgrid parameterizations. Such considerations may be relevant to other fluid dynamical systems such as plasmas and astrophysical objects.
One can perhaps imagine a supercomputer of the future ${ }^{96}$, geared towards such fluid dynamical computations of nonlinear multiscale systems more generally. As with present-day supercomputers, such a supercomputer would contain multiple cabinets, that is, clusters of computing nodes. These cabinets would be grouped into 'constellations'. The higher the constellation number, the more cabinets it would contain. The power consumed by each constellation would be roughly the same. The first constellation would be for computations and for systems software in which output must be precise. The second constellation would comprise multiple cabinets in which the processors were not quite precise and in which data are transported at reduced precision. For the third constellation, imprecision would be further increased, and so on. Such architecture may already arise in brains thus helping explain why it is possible to operate the 80 billion neurons in human brains with just $20 \mathrm{~W}$ of power ${ }^{97}$.

\section{Outlook}

I have argued on both theoretical and practical bases that all comprehensive weather and climate models, no matter how fine their resolution, should be stochastic in nature. Of course, much research remains to be done. At the practical end, work is ongoing to ensure conservation properties are properly respected in schemes such as SPPT; at the theoretical end, work continues to develop rigorous stochastic parameterizations from first principles. As discussed, there is considerable scope for using the methods of artificial intelligence to derive computationally efficient reducedprecision stochastic parameterizations, a considerable growth area for the future.

\section{T. N. Palmer(D)}

Department of Physics, University of Oxford, Oxford, UK.

e-mail: tim.palmer@physics.ox.ac.uk https://doi.org/10.1038/s42254-019-0062-2

Published online: 30 May 2019

1. IPCC Climate Change 2013: The Physical Science Basis (eds Stocker, T. F. et al.) (Cambridge Univ. Press, 2013).

2. IPCC Climate Change 2014: Impacts, Adaptation, and Vulnerability. Part A: Global and Sectoral Aspects (eds Field, C. B. et al.) (Cambridge Univ. Press, 2014).

3. Shepherd, J. G. et al. Geoengineering the Climate: Science, Governance and Uncertainty (The Royal Society Publishing, 2009).

4. Sutton, R. B. et al. Attributing extreme weather to climate change is not a done deal. Nature 561, 177 (2018).

5. Suckling, E. in Weather and Climate Services for the Energy Industry (ed. Troccoli, A.) 123-137 (Palgrave Macmillan, 2018).

6. Cantelaube, P. \& Terres, J.-M. Seasonal weather forecasts for crop yield modelling in Europe. Tellus 57A, 476-487 (2004).
7. Thomson, M. C. et al. Malaria early warnings based on seasonal climate forecasts from multi-model ensembles. Nature 439, 576-579 (2006).

8. Palmer, T. N. The prediction of uncertainty in weather and climate forecasting. Rep. Prog. Phys. 63, 71-116 (2000).

9. Palmer, T. N. The ECMWF ensemble prediction system: looking back (more than) 25 years and projecting forward 25 years. Q. J. R. Meteorol. Soc. https:// doi.org/10.1002/qj.3383 (2018).

10. Wilks, D. Statistical Methods in the Atmospheric Sciences (Academic Press, 2011).

11. Weisheimer, A. \& Palmer, T. N. On the reliability of seasonal climate forecasts. J. R. Soc. Interface 11 20131162 (2014).

12. Houghton, J. The Physics of Atmospheres (Cambridge Univ. Press, 2002)

13. Hasselmann, K. Stochastic climate models. Part I. Theory. Tellus 28, 473-485 (1976)

14. Clement, A. K. et al. The Atlantic Multidecadal Oscillation without a role for ocean circulation. Science 350, 320-324 (2015)

15. Zhang, R. et al. Comment on "The Atlantic Multidecadal Oscillation without a role for ocean circulation". Science 352, 1527 (2016)

16. Palmer, T. N. A nonlinear dynamical perspective on model error: A proposal for non-local stochasticdynamic parameterization in weather and climate prediction. Q. J. R. Meteorol. Soc. 127, 279-304 (2001).

17. Palmer, T. N., Doering, A. \& Seregin, G. The real butterfly effect. Nonlinearity 27, R123-R141 (2014).

18. Arakawa, A. The cumulus parameterization problem: past, present, and future. J. Clim. 17, 2493-2525 (2004).

19. Palmer, T. N., Shutts, G. J. \& Swinbank, R. Alleviation of a systematic westerly bias in general circulation and numerical weather prediction models through an orographic gravity wave drag parameterization. O. J. R. Meteorol. Soc. 112, 1001-1031 (1986).

20. Gent, P. R. \& McWilliams, J. C. Isopycnal mixing in ocean circulation models. J. Phys. Oceanogr. 20, 150-155 (1990).

21. Neelin, J. D., Peters, O., Lin, J. W.-B., Hales, K. \& Holloway, C. E. Rethinking convective quasi-equilibrium observational constraints for stochastic convective schemes in climate models. Phil. Trans. R. Soc. A 366 2579-2602 (2008).

22. Majda, A. J. \& Bertozzi, A. L. Vorticity and Incompressible Flow (Cambridge Univ. Press, 2002).

23. Nastrom, G. D. \& Gage, K. S. A climatology of atmospheric wavenumber spectra observed by commercial aircraft. J. Atmos. Sci. 42, 950-960 (1985).

24. Lovejoy, S. \& Schertzer, D. The Weather and Climate (Cambridge Univ. Press, 2013).

25. Zhang, X., Liu, H. \& Zhang, M. Double ITCZ in coupled ocean-atmosphere models: from CMIP3 to CMIP5. Geophys. Res. Lett. 42, 8651-8659 (2015).

26. Masato, G., Hoskins, B. J. \& Woollings, T. Winter and summer Northern Hemisphere blocking in CMIP5 models. J. Clim. 26, 7044-7059 (2013).

27. Palmer, T. N. A personal perspective on modelling the climate system. Proc. R. Soc. A 472, 20150772 (2016).

28. Buizza, R., Miller, M. \& Palmer, T. N. Stochastic representation of model uncertainties in the ECMWF ensemble prediction system. Q. J. R. Meteorol. SoC 125, 2887-2908 (1999)

29. Palmer, T. N. et al. Stochastic Parametrization and Model Uncertainty ECMWF Technical Memoranda (ECMWF, 2009); http://www.ecmwf.int/sites/default/ files/elibrary/2009/11577-stochasticparametrization-and-model-uncertainty.pd

30. Majda, A. J., Timofeyev, I. \& Vanden Eijnden, E. A mathematical framework for stochastic climate models. Commun. Pure Appl. Math. 54, 891-974 (2001).

31. Palmer, T. N. Towards the probabilistic Earth-system simulator: a vision for the future of climate and weather prediction. Q. J. R. Meteorol. Soc. 138, 841-861 (2012)

32. Holm, D. D. Variational principles for stochastic fluid dynamics. Proc. R. Soc. A 471, 20140963 (2015).

33. Plant, R. S. \& Craig, G. C. A stochastic parameterization for deep convection based on equilibrium statistics. J. Atmos. Sci. 65, 87-105 (2008).

34. Bengtsson, L., Steinheimer, M., Bechtold, P. \& Geleyn, J.-F. A stochastic parametrization for deep convection using cellular automata. Q. J.R Meteorol. Soc. 139, 1533-1543 (2013). 
35. Gottwald, G. A., Peters, K. \& Davies, L. A data-driven method for the stochastic parametrisation of subgridscale tropical convective area fraction. Q. J. R. Meteorol. Soc. 142, 349-359 (2016)

36. Khouider, B., Biello, J. \& Majda, A. J. A stochastic multicloud model for tropical convection. Commun. Math. Sci. 8, 187-216 (2010).

37. Tompkins, A. M. $\&$ Berner, J. A stochastic convective approach to account for model uncertainty due to unresolved humidity variability. J. Geophys. Res. 113, D18101 (2008).

38. Berner, J. et al. Stochastic parameterization: toward new view of weather and climate models. Bull. Am. Meteorol. Soc. 98, 565-587 (2017).

39. Mason, P. J. \& Thomson, D. J. Stochastic backscatter in large-eddy simulations of boundary layers. J. Fluid. Mech. 242, 51-78 (1992).

40. Shutts, G. A kinetic energy backscatter algorithm for use in ensemble prediction systems. Q. J. R. Meteorol. Soc. 131, 3079-3102 (2005).

41. Berner, J., Shutts, G. J., Leutbecher, M. \& Palmer, T. N. A spectral stochastic kinetic energy backscatter scheme and its impact on flow-dependent predictability in the ECMWF ensemble prediction system. J. Atmos. Sci. 66 603-626 (2009)

42. Palmer, T. N. \& Williams, P. Stochastic Physics and Climate Modelling (Cambridge Univ. Press, 2017).

43. Wouters, J. \& Lucarini, V. Disentangling multi-level systems: averaging, correlations and memory. J. Stat. Mech. 3, P03003 (2012).

44. Wouters, J. \& Lucarini, V. Multi-level dynamical systems: connecting the Ruelle response theory and the Mori-Zwanzig approach. J. Stat. Phys. 151, 850-860 (2013)

45. Franzke, C. L., O'Kane, T. J., Berner, J., Williams, P. D. $\&$ Lucarini, V. Stochastic climate theory and modeling. WIREs Clim. Change 6, 63-78 (2015).

46. Vissio, G. \& Lucarini, V. A proof of concept for scaleadaptive parametrizations: the case of the Lorenz '96 model. Q. J. R. Meteorol. Soc. 144, 63-75 (2018).

47. Bengtsson, L.et al. A model framework for stochastic representation of uncertainties associated with physical processes in NOAA's Next Generation Global Prediction System (NGGPS). Mon. Weather Rev., https://doi.org/ 10.1175/MWR-D-18-0238.1 (2019).

48. Shutts, G. J. \& Palmer, T. N. Convective forcing fluctuations in a cloud-resolving model: relevance to the stochastic parameterization problem. J. Clim. 20, 187-202 (2007)

49. Shutts, G. J. \& Callado Pallarès, A. Assessing parameterization uncertainty associated with horizontal resolution in numerical weather prediction models. Phil. Trans. R. Soc. Lond. A 372, 20130284 (2014).

50. Christensen, H. M. Constraining stochastic parametrisation schemes using high-resolution simulations. Preprint at arXiv https://arxiv.org abs/1904.04503 (2019).

51. Christensen, H. M., Lock, S. J., Moroz, I. M. \& Palmer, T. N. Introducing independent patterns into the stochastically perturbed parametrization tendencies (SPPT) scheme. Q. J. R. Meteorol. Soc. 143, 2168-2181 (2017).

52. Chevallier, F., Chéruy, F., Scott, N. A. \& Chédin, A A neural network approach for a fast and accurate computation of longwave radiative budget. J. Appl. Meteorol. 37, 1385-1397 (1998).

53. Krasnopolsky, V. M. The Aapplication of Neural Networks in the Earth-system Sciences Atmospheric and Oceanographic Sciences Library, Vol. 46 (Springer, 2013).

54. Andrejczuk, M. et al. Oceanic stochastic parameterizations in a seasonal forecast system. Mon. Weather Rev. 144, 1867-1875 (2016).

55. Porta Mana, P. G. L. \& Zanna, L. Toward a stochastic parameterization of ocean mesoscale eddies. Ocean Model. 79, 1-20 (2014)

56. MacLeod, D. A., Cloke, H. L., Pappenberger, F. \& Weisheimer, A. Improved seasonal prediction of the hot summer of 2003 over Europe through better representation of uncertainty in the land surface. Q. J. R. Meteorol. Soc. 142, 79-90 (2016).
57. Juricke, S. \& Jung, T. Influence of stochastic sea ice parameterization on climate and the role of atmosphere-sea ice-ocean interaction. Phil. Trans. $R$. Soc. Lond. A 372, 20130283 (2014).

58. Williams, P. D. Climatic impacts of stochastic fluctuations in air-sea fluxes. Geophys. Res. Lett. 39, L10705 (2012).

59. Murphy, A. H. A note on the utility of probabilistic predictions and the probability score in the cost-loss ratio decision situation. J. Appl. Meteorol. 5 534-537 (1966)

60. Palmer, T. N. \& Richardson, D. Decisions, decisions... In ECMWF Newsletter 12-14 (ECMWF, 2014); https:/ www.ecmwf.int/sites/default/files/elibrary/2014/ 14584-newsletter-no141-autumn-2014.pdf

61. Weisheimer, A., Corti, S., Palmer, T. \& Vitart, F Addressing model error through atmospheric stochastic physical parametrizations: impact on the coupled ECMWF seasonal forecasting system. Phil. Trans. R. Soc. A 372, 20130290 (2014).

62. Subramanian, A., Weisheimer, A., Palmer, T., Vitart, F $\&$ Bechtold, P. Impact of stochastic physics on tropical precipitation in the coupled ECMWF model. Q. J. R. Meteorol. Soc. 143, 852-865 (2017).

63. Christensen, H. M., Berner, J., Coleman, D. \& Palmer, T. N. Stochastic parameterization and the El Niño-Southern Oscillation. J. Clim. 30, 17-38 (2017).

64. Strommen, K., Christensen, H. M., Berner, J. \& Palmer, T. N. The impact of stochastic parametrisation on the representation of the Asian summer monsoon. Clim. Dyn. 50, 2269-2282 (2018).

65. Mo, K. \& Ghil, M. Cluster analysis of multiple planetary flow regimes. J. Geophys. Res. 93, 10927-10952 (1988).

66. Weaver, M. Summer 2018 was UK's joint hottest on record, Met Office says. The Guardian https://www. theguardian.com/uk-news/2018/sep/03/summer2018-uk-joint-hottest-on-record-met-office-says (2018).

67. King, A. \& Henley, B. It's a savage summer in the Northern Hemisphere - and climate change is slashing the odds of more heatwaves. The Conversation https://theconversation.com/its-a-savage-summer-inthe-northern-hemisphere-and-climate-changeis-slashing-the-odds-of-more-heatwaves- 100582 (2018).

68. Heatwave in northern Europe, summer 2018. World Weather Attribution https://www. worldweatherattribution.org/attribution-of-the2018-heat-in-northern-europe/ (2018)

69. de la Hamaide, S., Devitt, P. \& Hogan, M. Heatwave ravages European fields, sending wheat prices soaring Reuters https://www.reuters.com/article/us-europewheat-harvest/heatwave-ravages-european-fieldssending-wheat-prices-soaring-idUSKBN 1 KNOL9 (2018).

70. Vaughan, A. UK summer 'wind drought' puts green revolution into reverse. The Guardian https://www. theguardian.com/environment/2018/aug/27/uksummer-wind-drought-puts-green-revolution-intoreverse (2018)

71. Woollings, T. et al. Blocking and its response to climate change. Curr. Clim. Change Rep. 4, 287-300 (2018).

72. Schiemann, R., Demory, M.-E., Shaffrey, L. C., Strachan, J. \& Vidale, P.-L. The resolution sensitivity of Northern Hemisphere blocking in four $25-\mathrm{km}$ atmospheric global circulation models. J. Clim. 30, 337-358 (2017)

73. Green, J. S. A. The weather during July 1976: some dynamical considerations of the drought. Weather 32 120-126 (1977)

74. Dawson, A. \& Palmer, T. N. Simulating weather regimes: impact of model resolution and stochastic parameterization. Clim. Dyn. 44, 2177-2193 (2015).

75. Lorenz, E. N. Deterministic non-periodic flow. J. Atmos. Sci. 20, 130-141 (1963).

76. Kwasniok, F. Enhanced regime predictability in atmospheric low-order models due to stochastic forcing. Phil. Trans. R. Soc. A 372, 20130286 (2014).

77. Charney, J. G. \& DeVore, J. G. Multiple flow equilibria in the atmosphere and blocking. J. Atmos. Sci. 36, 1205-1216 (1979).
78. Kondrashov, D., Ide, K. \& Ghil, M. Weather regimes and preferred transition paths in a three-level quasigeostrophic model. J. Atmos. Sci. 61, 568-587 (2004).

79. Lorenz, E. N. in Predictability of Weather and Climate (eds Palmer, T. N. \& Hagedorn, R.) 40-58 (Cambridge Univ. Press, 1996)

80. Christensen, H. M., Moroz, I. M. \& Palmer, T. N. Simulating weather regimes: impact of stochastic and perturbed parameter schemes in a simple atmospheric model. Clim. Dyn. 44, 2195-2214 (2015).

81. Strommen, K. \& Palmer, T. N. Signal and noise in regime systems: a hypothesis on the predictability of the North Atlantic Oscillation. O. J. R. Meteorol. Soc. 145, 147-163 (2019)

82. Eade, R. et al. Do seasonal-to-decadal climate predictions underestimate the predictability of the real world?. Geophys. Res. Lett. 41, 5620-5628 (2014).

83. Palmer, T. N. Build high-resolution global climate models. Nature 515, 338-339 (2014)

84. Palmer, T. N. More reliable forecasts with less precise computations: a fast-track route to cloud-resolved weather and climate simulators. Phil. Trans. R. Soc. A 372, 20130391 (2014).

85. Văňa, F. et al. Single precision in weather forecasting models: an evaluation with the IFS. Mon. Weather Rev. 145, 495-502 (2017).

86. Dueben, P. D. \& Palmer, T. N. Benchmark tests for numerical weather forecasts on inexact hardware. Mon. Weather Rev. 142, 3809-3829 (2014).

87. Hatfield, S., Subramanian, A., Palmer, T. \& Düben, P. Improving weather forecast skill through reducedprecision data assimilation. Mon. Weather Rev. 146, 49-62 (2018).

88. Dawson, A., Düben, P. D., MacLeod, D. A. $\delta$ Palmer, T. N. Reliable low precision simulations in land surface models. Clim. Dyn. 51, 2658-2666 (2018).

89. Jeffress, S., Düben, P. \& Palmer, T. Bitwise efficiency in chaotic models. Proc. R. Soc. A 473, 20170144 (2017).

90. Thornes, T., Düben, P. \& Palmer, T. A power law for reduced precision at small spatial scales: experiments with an SQG model. Q. J. R. Meteorol. Soc. 144, 1179-1188 (2018).

91. Chantry, M. Thornes, T. \& Palmer, T. N. Scale-selective precision for weather and climate forecasting. Mon. Weather Rev. 147, 645-655 (2019).

92. Dueben, P. D., Russell, F. P., Niu, X., Luk, W. $\&$ Palmer, T. N. On the use of programmable hardware and reduced numerical precision in earth-system modeling. J. Adv. Model. Earth Syst. 7, 1393-1408 (2015).

93. Subramanian, A., Juricke, S., Dueben, P. \& Palmer, T. N. A stochastic representation of sub-grid uncertainty for dynamical core development. Bull. Am. Meteorol. Soc. https://doi.org/10.1175/BAMS-D-17-0040.1 (2019).

94. Palem, K. V. Energy aware computing through probabilistic switching: a study of limits. IEEE Trans. Comput. 54, 1123-1137 (2005)

95. Palem, K. V. Inexactness and a future of computing. Phil. Trans. R. Soc. A 372, 20130281 (2014).

96. Palmer, T. N. Modelling: build imprecise supercomputers Nature 526, 32-33 (2015).

97. Palmer, T. N. \& O'Shea, M. Solving difficult problems creatively: a role for energy optimised deterministic/ stochastic hybrid computing. Front. Comput. Neurosci. 9, 124 (2015)

\section{Acknowledgements}

The author thanks H. Christensen for helpful input on and improvements to this paper. This work was supported by the ERC Advanced Grant ITHACA grant number DCR00620.

\section{Competing interests}

The author declares no competing interests.

\section{Publisher's note}

Springer Nature remains neutral with regard to jurisdictional claims in published maps and institutional affiliations. 